\title{
MÁS QUE SONIDO: INTERPRETACIÓN DE MÚSICA INSTRUMENTAL EN LENGUA DE SIGNOS PARA LAS PERSONAS SORDAS
}

\section{More than sound: signed instrumental music for deaf people}

\author{
AliCIA PEÑAlBA ACITORES; CARLOS MORIYÓN MOJICA; SONIA LUQUE PEREA \\ FACULTAD DE EDUCACIÓN \\ Avd. Madrid, 50, 34004 Palencia \\ alicia.penalba@uva.es \\ ORCID: http://orcid.org/0000-0002-5725-0639 \\ Recibido: 31/12/ 2017/ Aceptado: 15/03/2018 \\ Cómo citar: Peñalba, A., Moriyón, C., \& Luque, S. (2018). Más que sonido: interpretación de música \\ instrumental en lengua de signos para las personas sordas. Tabanque: Revista Pedagógica, 31. P. 94- \\ 107 \\ DOI: https://doi.org/10.24197/trp.31.2018.94-107
}

Resumen: Las personas sordas son uno de los colectivos que menor interés presenta hacia la música debido a que históricamente se ha resaltado su componente auditivo. No obstante, investigaciones recientes se interesan por la música como fenómeno multimodal. Esta posibilidad permite hacer accesible la música a personas sordas. En este artículo se abordan varios proyectos de música para/con sordos y se analizan las posibilidades de la interpretación musical en lengua de signos de la música instrumental pura.

Palabras clave: Música en lengua de signos; metáforas cognitivas; música instrumental; proyectos musicales para sordos; cognición musical corporeizada

\begin{abstract}
Deaf people are one of the groups that show the least interest in music because its auditory component has been historically highlighted. However, recent research is interested in music as a multimodal phenomenon. This possibility allows to make music accessible to deaf people. In this article several music projects for/with deaf people are addressed and the possibilities of musical interpretation in sign language of pure instrumental music are analyzed.
\end{abstract}

Keywords: Music in Signed Language; cognitive metaphor; instrumental music; music projects for deaf;

Sumario: 1. Música y personas sordas: algunos proyectos musicales; 2 . Interpretación de música través de metáforas cognitivas; 3. Propuesta de interpretación instrumental. El carnaval de los Animales; 4. Conclusiones

Summary: 1. Music and deaf: some musical projects; 2. Interpretation of music through cognitive metaphors; 3. Proposal of instrumental music interpretation: The Carnival of Animals; 4. Conclusion 
En su concepción más tradicional, la música se considera un arte eminentemente auditivo cuya forma de expresión se materializa a través del sonido. Aunque el fenómeno sonoro tiene una vital importancia en esta manifestación artística, otras modalidades perceptivas están implicadas en el disfrute de la música. En los últimos años están surgiendo estudios que reformulan la consideración de esta y la defienden como un fenómeno multimodal que trasciende lo sonoro. Se contemplan con esta nueva perspectiva la importancia de la imagen (Platz \& Kopiez, 2012; Thompson, Graham, \& Russo, 2005), la relevancia de las sensaciones táctiles (Malamud-Kessler, Estañol-Vidal, Ayala-Anaya, SentíesMadrid, \& Marco, 2014) y la percepción y conceptualización del movimiento (Soria-Urios, Duque, \& García-Moreno, 2011) como algo consustancial al fenómeno sonoro-musical.

Una de las consecuencias del tratamiento fundamentalmente auditivo que ha recibido la música a lo largo de la historia es que las personas sordas son quizás el colectivo que menor interés manifiesta con respecto a ella. No obstante, en la actualidad la música para las personas sordas constituye un tema de interés académico creciente (Schraer-Joiner, 2014) y que se ocupa del rol que desempeña la música en la cultura sorda (Darrow and Loomis 1999; Darrow 2007), o de la ampliación del lenguaje musical hacia aspectos hápticos y motrices (Nanayakkara, Taylor, Wyse, \& Ong, 2009)

\section{MÚSICA Y PERSONAS SORDAS: ALGUNOS PROYECTOS MUSICALES}

En los últimos años, agrupaciones nacionales e internacionales se comienzan a interesar por hacer accesible la música a las personas sordas. Los grupos Handmade (danés), Talking Hands (americano), D-Pan (americano), SPIT o SHAPE (ingleses), SignMark (finlandés), Kwan Yin (chino) y Sia (estadounidense) son algunas de las manifestaciones internacionales de artistas interesados por la performance musical en lengua de signos (Peñalba, Moriyón, Luque, \& Cabezas, 2017). El proyecto Signed Music Project, del que se hablará más adelante, propone un listado de intérpretes, desde los años 30 del siglo pasado ${ }^{1}$, de música en lengua de signos que incluye algunos artistas relevantes de la última década como los que se proponen a continuación junto con un extracto de su producción artística:

Sanborn, I. (2014). Another Fly Fish Story (American Sign Language).

Click here for the performance

Timm, R. L. (2014). Tell Your Story.

Click here for the performance

Gaina, E. (2015). Eye Music Captures the True Essence of the Human Spirit.

${ }^{1}$ http://wp.towson.edu/signedmusic/chronological-signed-music-performances/ 


\section{Click here for the performance \\ Virnig, D. (2015). Fish. \\ Click here for the performance \\ The Fenicle Brothers. (2015). Food Chain. \\ Click here for the performance \\ Witcher, P. (2015). Nice and Slow. \\ Click here for the performance \\ Cripps, J. H. (2017). Rain. \\ Click here for the performance}

En España, los colectivos Ojos que oyen (Madrid), ILSEVIN (Asturias), Arymux, el proyecto Signos a Escena, el Coro Bilingüe del Centro Educativo Ponce de León, o intérpretes como Beatriz Romero (con Rozalén) o Gonzalo Cabezas (2013) han presentado propuestas de acercamiento de la música a los sordos con diversas aproximaciones metodológicas. Una gran parte de estas interpretaciones se basa en el contenido del texto y apenas existen propuestas de interpretación de música instrumental.

En la actualidad, el interés por estudiar la relación entre la música y las personas sordas ha dado lugar a diversos proyectos de investigación con orientaciones muy diferentes. Algunos tratan de estudiar los beneficios del entrenamiento musical en personas con discapacidad auditiva, otros tratan de mejorar la accesibilidad a través de diversos medios, y otros de dotar de herramientas creativas a los propios sordos para que desarrollen su idea de música en lengua de signos. Algunos de los principales proyectos son los siguientes:

\section{Sounding Out: Music with hearing impaired children}

Este proyecto de investigación, de tres años de duración (2015-2018) en colaboración con la UCL Ear Institute y financiado por Creative Futures investiga el impacto de la actividad musical en adolescentes con discapacidad auditiva de entre 12 y 14 años en dos institutos de Londres. Participan Graham F Welch, Jo Saunders, Sian Edwards, Zoe Palmer, Evangelos Himonides, Julian Knight, Merle Mahon, Susanna Griffin and Deborah A Vickers y muestran los beneficios del entrenamiento musical en este colectivo.

https://www.researchgate.net/project/Sounding-Out-Music-with-hearing-impairedchildren

\section{Music Visualization Project}

Este proyecto estudia las posibilidades de varias ayudas técnicas que favorezcan la visualización sonora a través del uso de la tecnología. Se analizan, entre otros, el Music Animation Machine o el iTunes visualizer. El director del 
proyecto es David W. Fourney, del Centre for Learning Technologies Ryerson University Toronto, Canadá.

https://www.researchgate.net/project/Music-Visualization-for-the-Deaf-and-Hardof-Hearing

\section{Signed Music Project}

Este proyecto explora la música signada como una forma de expresión artística que surge en la comunidad sorda y que evoluciona de la poesía en lengua de signos y de la interpretación de las letras de las canciones. Este proyecto, liderado por Cripps \& Small ha sido presentado a la convocatoria del "Deaf culture centre” del Canadian Council for the Arts en 2014. Recopila interpretaciones musicales hechas por sordos y otros materiales valiosos para que el desarrollo de esta manifestación artística sea posible en esta comunidad.

http://wp.towson.edu/signedmusic/contact/

\section{Sign \& Sing. Reimagining Art Song Interpretation with American Sign Language}

Este proyecto refuerza la interpretación de música fundamentalmente clásica utilizando poemas o relatos. Se interpreta música de Leonard Bernstein, Frank Bridge, Aaron Copland, y Ricky Ian Gordon con poesía de Mathew Arnold, Emily Dickinson, Heinrich Heine, Langston Hughes, Percy Bysshe Shelley, entre otros.

https://www.symphonyspace.org/event/9218/Music/sign-sing

\section{Feel the Music}

Este proyecto forma parte de la Mahler Chamber Orchestra con el ciclo de conciertos denominado "Beethoven Journey". Son conciertos de música de este compositor pensados para ser experimentados por niños sordos a través de todos los sentidos. Los niños se sitúan en el centro de la orquesta, perciben las vibraciones, tocan los instrumentos, dirigen a los músicos, y ven la música. Han participado en esta experiencia tres escuelas de Zurich y Lucerna.

https://www.youtube.com/watch?v=CDpE1ajC4mo

\section{El lenguaje de la danza que surge del silencio}

El proyecto concedido al amparo de la convocatoria "Arte para la mejora social 2013" de la Obra Social "La Caixa”, se centra en el desarrollo creativo de personas con discapacidad auditiva a través de la danza de la India con el objetivo de mejorar su autoconocimiento e integración social. En las artes escénicas de la India, se considera la expresión gestual (que incluye la mano o mudra, y la expresión facial y corporal) como el principal elemento de comunicación a través del cual podemos crear poesía visual, narrar historias, y sobre todo, expresar emociones que, según la teoría estética de la India, es la finalidad del arte. Existen 
entre la lengua de signos de las personas sordas y la danza de la India numerosos puntos en común que sirven de puente para aplicar algunos de los principios de la danza a esta lengua a través de un trabajo creativo. De este modo, la persona sorda, utilizando su propia lengua, puede investigar en un ámbito poco desarrollado en la cultura sorda de nuestro país como es la capacidad de expresión artística a través de danza, la música y la poesía.

http://monicadelafuente-danza.blogspot.com.es/2014/06/el-lenguaje-de-la-danzaque-surge-del.html

\section{Propuesta de inTERPRetación de música a travÉS de METÁforas COGNITIVAS}

La interpretación de música instrumental en lengua de signos entraña numerosas dificultades. La primera de ellas es la derivada de la inexistencia de algunos signos referidos al fenómeno sonoro que la propia lengua no ha desarrollado por la dificultad de las personas sordas de percibir y, por tanto, de conceptualizar el fenómeno musical. Algunos de los signos musicales que se utilizan son el resultado de un trasvase literal de la cultura oyente, por lo que no es posible precisar si coinciden con las formas de percibir y conceptualizar dichos fenómenos por los propios sordos. El trabajo de Cabezas incluye algunas propuestas de interpretación de cualidades del sonido. Tal es el caso de la altura, utilizando el espacio de signación; la intensidad, utilizando los modificadores gestuales; y la duración, con la velocidad sígnica (Cabezas, 2013; p.33-43). Estas aportaciones pueden suponer un gran avance en este sentido, aunque se mantienen en la conceptualización de parámetros sonoros propios de la cultura oyente.

Otro de los problemas es el derivado de la multimodalidad del lenguaje musical que incluye aspectos puramente sonoros, pero otros gestuales, motrices, visuales, metafóricos, interpretativos, etc. Esto implica que a la hora de tomar decisiones sobre la interpretación sea necesario resaltar determinados estímulos y obviar otros. En la música instrumental acontecen además diversos fenómenos sonoros simultáneamente: ritmos, instrumentación, melodías, repeticiones, fraseos, silencios, etc. Por todas estas razones, la interpretación de música en lengua de signos se convierte en un fenómeno altamente subjetivo.

Amber Gallowy-Gallego es una intérprete de lengua de signos preocupada por la parte musical de las interpretaciones. Hace una propuesta de interpretación de música vocal e instrumental utilizando intuitivamente algunas metáforas clásicas como la de la verticalidad para la altura musical, o la densidad a través de la expresión facial. Se puede consultar una entrevista de gran interés en este enlace: https://www.youtube.com/watch?v=EuD2iNVMS_4 
Otra intérprete, Holly Maniatty lleva a cabo interpretaciones de diversos grupos musicales, desde hip-hop hasta pop o rock, como U2 o Bruce Springsteen también a través de usos intuitivos del lenguaje.

https://www.youtube.com/watch?v=19Tzlvl2tbg

Una de las posibilidades de llevar a cabo interpretaciones de música instrumental en lengua de signos parte del uso de metáforas cognitivas, ya que están presentes en esta lengua, fundamentalmente en los contextos más creativos (poemas, cuentos, teatro, etc). Las metáforas cognitivas son formas de conceptualización que utilizan las características de un dominio cognitivo conocido, para comprender otro más lejano. La esencia de la metáfora es "entender y experimentar un tipo de cosa en términos de otra” (Lakoff \& Johnson, 1980, p. 41). Para estos autores, "lo que ocurre es, simplemente, que el concepto se estructura metafóricamente, la actividad se estructura metafóricamente y, en consecuencia, el lenguaje se estructura también metafóricamente” (pp.41-42). Algunos autores se han sentido interesados por las posibilidades que el sistema lingüístico viso-gestoespacial ofrece al fenómeno de conceptualización metafórica en LSE (Moriyón et al., 2010).

El estudio de las metáforas en lengua de signos ha sido abordado de forma profunda por diversos autores (Fernández-Viader, Moriyón, Codorniu i Tomás, \& Valdespino, 2005; Carlos Moriyón, Fernández-Viader, \& Codorniu i Tomás, 2006). Algunas de las más representativas que se recogen en Peñalba et al., (2017, pp. 9394) son las siguientes:

a) metáforas estructurales (el conocimiento es visión / las ideas son energía / ...) b) metáforas orientacionales (abstracto es arriba - concreto es abajo / bueno es arriba malo es abajo / futuro es delante - pasado es detrás / más es arriba - menos es abajo / moral es arriba / poder es arriba / status es arriba / ...) c) metáforas ontológicas (el cuerpo es un objeto -y un contenedor- / la mente es un objeto -y un contenedor/ las ideas son objetos ${ }^{3} / \ldots$ ), muchas de las cuales, como ha quedado demostrado ya en distintos trabajos, son compartidas también por la LSE y la LSC, las dos lenguas de signos españolas.

Las personas oyentes utilizamos constantes metáforas en la vida cotidiana (George Lakoff \& Johnson, 1983) y en la conceptualización musical con más motivo, pues la música se caracteriza por su componente abstracto. Por esta razón se considera que las metáforas pueden constituir un punto de anclaje para la

\footnotetext{
${ }^{2}$ A propósito de esta metáfora conceptual y su relación con la de 'bueno es arriba' en la ASL, vid., entre otros, Sweetser (1995).

${ }^{3} \mathrm{Y}$, en tanto objetos, pueden ser manipulados o recolocados, pueden ser asidos o agarrados y pueden ser discriminados y elegidos.
} 
interpretación musical ya que los significados emergen del complejo sonorokinético-corporal (Shifres, 2007). El componente no sonoro es fundamental para los sordos y, gracias a la metáfora, se puede conceptualizar en términos de un dominio conocido, otro más remoto para ellos (Cox, 2001, 2011).

Una de las metáforas más comunes referidas por oyentes, que se extiende incluso al lenguaje hablado, es la que permite la comprensión del tono a través del esquema de verticalidad (agudo es arriba / grave es abajo). Feld (1985) identifica varias metáforas cognitivas en la música de los Kaluli de Nueva Guinea y establece relaciones estructurales entre las cascadas de agua y una melodía caracterizada por un descenso melódico (Peñalba et al., 2017). El mismo esquema de verticalidad permite conceptualizar los "lamentos" como descensos (Marconi, 2001). El esquema arriba-abajo se aplica fundamentalmente a la altura, como muestran los trabajos de varios teóricos (Echard, 1999, 2006; Saslaw, 1996; Zbikowski, 2011). La conceptualización del tono en el espacio lleva acompañando a diversas manifestaciones de la cultura musical occidental desde que la música se escribe. La notación musical del canto gregoriano representa las ayudas nemotécnicas que se utilizaban para recordar los ascensos y descensos de la música a través de gestos con las manos.

Otros esquemas clásicos utilizados en la conceptualización musical incluyen el esquema ciclo para la comprensión de la modulación armónica; cercalejos para comprender la cercanía tonal (Saslaw, 1996); el esquema centro-periferia para los grados tonales (Jacquier \& Callejas Leiva, 2013); o el esquema atracción para comprender la centralidad tonal (Johnson \& Larson, 2003).

La heterogeneidad de los estudios invita a la reflexión sobre la verdadera naturaleza del esquema encarnado y la proyección metafórica. El esquema ha de ser estrictamente de carácter sensoriomotor y para ello se cuenta con el source domain y el target domain o, lo que es lo mismo, el esquema encarnado y el fenómeno abstracto que se comprende a través de proyecciones metafóricas (Peñalba, 2005).

\section{PRopuesta de interpretación InSTRUMENTAL. EL CARNAVAl DE LOS ANIMALES}

Se analiza aquí el trabajo presentado por Peñalba, Moriyón, Luque, \& Cabezas (2015) de propuesta de interpretación de la Suite Musical "Le carnaval des animaux" del compositor romántico francés Camille Saint-Saëns, obra instrumental que está creada especialmente para niños. 
La propuesta hace hincapié en algunas metáforas cognitivas y otros usos expresivos de la lengua de signos, que se ejemplificarán a través del primer movimiento (Introduction et Marche royale du lion. Andante maestoso. Para 2 pianos, 2 violines, viola, violonchelo y contrabajo) aunque se pueden visualizar todos los movimientos en los siguientes enlaces:

1.- Introducción y marcha real del león. Andante maestoso http://youtu.be/dBFPb3M58K8

2.- Gallinas y gallos. Allegro moderato

http://youtu.be/b6f7vo204W0

3.- Hemíonos (los asnos salvajes del Tíbet). Presto

furioso http://youtu.be/ZwrRMYyrjh4

4.- Tortugas. Andante maestoso

http://youtu.be/bYSORdHnW3Q

5.- El elefante. Allegretto pomposo

http://youtu.be/16-Tk552ocY

6.- Canguros. Moderato

http://youtu.be/5kyZeMxsjDA

7.- Aquarium. Andantino

http://youtu.be/ko3yY3CJOkM

8.- Personajes de largas orejas. Tempo ad

libitum http://youtu.be/RIRJom_K5jU

9.- El cuclillo en el fondo del bosque.

Andante http://youtu.be/vjdLpONG7kQ

10.- Pajarera. Moderato grazioso

http://youtu.be/HokwmX4oj5M

11.- Pianistas. Allegro moderato

http://youtu.be/VtH7YEW5-5I

12.- Fósiles. Allegro ridicolo

http://youtu.be/GvKc6vPinN0

13.- El cisne. Andantino

gracioso https://www.youtube.com/watch?v=0d-QIX0WVcI

14.- Final. Molto

Allegro https://www.youtube.com/watch?v=HXf-inDy3Xc

Como se ha mencionado con anterioridad, la música trasciende el fenómeno sonoro y además del sonido otros elementos implicados en la percepción musical pueden ser relevantes para las personas sordas. Para ello esta propuesta incorpora aspectos musicales y otros extramusicales como visuales o vibrotáctiles y kinestésicos. 


\subsection{Aspectos musicales}

\section{Presentación de los instrumentos}

La presentación de los instrumentos y su forma de ejecución, previa al comienzo de la pieza musical, será de ayuda para comprender los aspectos tímbricos y de carácter de la pieza ${ }^{4}$. En ocasiones los instrumentos pueden volver a aparecer de nuevo durante el transcurso de la música. Las cualidades tímbricas se explicitan por medio de la expresión facial y corporal (Peñalba et al., 2017, p. 101).

En el caso que nos ocupa, el violín y la viola se interpretarán a través de un movimiento similar. Las diferencias tímbricas entre ambos instrumentos se comunicarán mediante componentes no manuales -quinésica facial-: el fruncimiento del ceño, la abertura de los ojos, y el carácter labiodental -la mordida del labio inferior-, que permitirán representar el timbre más grave de la viola frente al del violín. El violonchelo y el contrabajo se diferenciarán, a nivel gestual, por el tamaño, pero también porque la presentación del contrabajo se acompañará de una gestualidad facial similar a la establecida para la viola (ceño fruncido, ojos muy abiertos y labio inferior mordido). Dado que en estos casos los instrumentos se pueden tocar en pizzicato, además de la gestualidad propia de éste, se acompañará de la fonación correspondiente a la articulación fonémica de /pa-pa-pa/.

\section{Forma musical}

Es preciso representar gestualmente la estructura o forma de la pieza. Esto se hará a través de la repetición en las partes iguales o similares. En el movimiento que se analiza aquí la forma presenta una estructura AB-coda, que la profesoraintérprete representa, a nivel gestual, haciendo uso de un material corporal similar en cada una de las partes que contrastan entre sí.

\section{Pulso y acento}

Aunque la lengua de signos presenta una caracterización rítmica del signo (sobre todo en contextos creativos como la poesía), la propuesta que aquí se analiza implica la ejecución sígnica con ritmo medido. En la pieza se utilizan tanto signos manuales como clasificadores y ambos se sincronizan con el pulso musical, remarcando los acentos.

\section{Frase musical}

La frase musical muestra una entidad con significado propio, que en ocasiones se repite. La frase se representa a través de la lateralización del cuerpo o a través de las respiraciones. Es preciso que corporalmente exista una coherencia y una continuidad del movimiento durante toda la frase, para dotarla de entidad propia.

\footnotetext{
${ }^{4}$ Se puede observar dicha presentación en la interpretación de la Introducción y Marcha real del león https://www.youtube.com/watch?v=ne-uQKzHAXU 


\section{Metáforas cognitivas}

Algunas de las metáforas cognitivas utilizadas en este primer movimiento son las siguientes:

\section{La melodía es un camino}

Se representa al león en una especie de desfile hacia delante. La melodía es el camino que recorre el león y que se ve interrumpido por otros personajes, cuando la melodía se detiene. La música en este caso, que recuerda a una fanfarria de trompetas, interpretada por el piano, le concede un carácter solemne que se representa a través de la expresión facial y corporal. El camino recorrido por el león se extiende a lo largo de todo el movimiento, por lo que se hace coincidir el final de la obra con el final del camino.

\section{El sonido es movimiento}

Una de las metáforas más básicas e intuitivas que se utilizan consiste en la relación entre sonido y movimiento. El movimiento no comienza hasta que la música comienza a sonar y éste para cuando llega el silencio. También se representa el sonido ordenado con patrones reconocibles y el sonido difuso con patrones menos reconocibles. Los pasos del león son definidos, frente a la llegada de los animales que se presenta con una gestualidad difusa y, en este sentido, el sonido es movimiento, pero un tipo de movimiento determinado en función de la sonoridad.

\section{La intensidad sonora es vibración}

Las personas sordas se percatan del sonido, en gran medida, por la vibración que perciben a través de los mecanorreceptores de su piel. En lengua de signos, algunos sonidos fuertes como sonar el teléfono o roncar se realizan a través de signos en movimiento vibratorio. Los rugidos del león (caracterizado en la música como escalas cromáticas ascendentes y descendentes interpretadas en forte y con sonoridad predominantemente grave) se representan con movimiento vibratorio.

\section{El tono es espacio}

El uso del espacio para la metaforización en lengua de signos es algo muy recurrente en la lengua, como se ha especificado anteriormente: abstracto es arriba concreto es abajo / bueno es arriba - malo es abajo / futuro es delante - pasado es detrás / más es arriba - menos es abajo / moral es arriba / poder es arriba / status es arriba /. Además, en música, la verticalidad constituye uno de los esquemas más básicos para conceptualizar la altura musical, como se ha mencionado previamente. En el caso que nos ocupa, el rugido del león se presenta en el eje vertical, con un ligero desplazamiento en diagonal. Las miradas de los animales en el paseo del león están también representadas espacialmente en el plano vertical. 


\section{La música es un ser animado}

Se produce en este sentido una personificación de la música. La música tiene una emoción determinada. En el caso de esta introducción, el carácter es solemne, pero con matices jocosos y humorísticos. La música es el león y adquiere, por tanto, sus características.

\subsection{Aspectos extramusicales}

Además de los elementos estrictamente musicales se introducen otras ayudas para mejorar la expresividad. Algunas de ellas son las siguientes:

Reforzamiento vibro táctil: En el momento de la escucha, las personas sordas pueden sostener un globo hinchado para percibir las vibraciones, que serán distintas en función de la altura, la textura sonora, etc. Este fue el caso de la interpretación de esta obra en el VI Festival de Canción en Lengua de Signos, celebrado en Alicante el 21 de enero de 2017.

Ampliación del espacio de signación: se han podido observar contextos en lengua de signos en los que el espacio de signación se amplía a prácticamente todo el cuerpo y se usan más los planos laterales y superior, como las interpretaciones hechas por Amber Gallowy-Gallego y Holly Maniatty.

Uso de elementos complementarios: en ocasiones puede ser de interés incluir elementos como imágenes, para reforzar el significado simbólico, o ventiladores, para ofrecer sensaciones de estar bajo el agua, como se muestra en el ejemplo "Aquarium" 5

\section{CONCLUSIONES}

La música es un fenómeno multimodal y, contemplado de esta manera, permite acercarlo a las personas sordas a través del énfasis en la gestualidad, la sensorialidad y el uso metafórico. Las metáforas empleadas para conceptualizar fenómenos musicales son producto de metaforizaciones de la propia lengua de signos, con lo que se trata de evitar las imposiciones propias de las conceptualizaciones musicales de los oyentes. La interpretación de música instrumental supone un reto que implica, además del uso de metáforas, otros elementos como el espacio de signación o el ritmo, cercanos a la poesía en lengua de signos, forma artística que surge dentro de la propia comunidad sorda. Es preciso estudiar en mayor profundidad las manifestaciones musicales que surgen de los

\section{${ }^{5}$ http://youtu.be/ko3yY3CJOkM}


propios sordos, para comprender sus formas de conceptualización y de ahí extraer más claves para las propuestas de interpretación musical.

Esta forma de interpretación de la música instrumental no solo supone una puerta abierta para acercarla a las personas sordas, sino que los oyentes también pueden servirse de ella como guía para la escucha, pues favorece su comprensión desde diversas perspectivas, incluyendo la sonora a través del cuerpo. En este sentido, las exigencias que la interpretación de música a lengua de signos hace recaer sobre el intérprete torna necesario replantear su figura, hasta acercarla a la del intérprete-performer y a las necesidades de formación y especialización que ello implica.

\section{BIBLIOGRAFÍA}

Cox, A. (2001). The mimetic hypothesis and embodied musical meaning. Musicae Scientiae, 5(2), 195-212.

Cox, A. (2011). Embodying music: Principles of the mimetic hypothesis. Music Theory Online, 17(2).

Darrow, A.-A. (2007). The Role of Music in Deaf Culture: Implications for Music Educators. Journal of Research in Music Education, 41(2), 93. http://doi.org/10.2307/3345402

Darrow, A. A., \& Loomis, D. M. (1999). Music and deaf culture: Images from the media and their interpretation by deaf and hearing students. Journal of Music Therapy, 36(2), 88-109. http://doi.org/10.1093/jmt/36.2.88

Echard, W. (1999). An analysis of Neil Young's "Powderfinger"based on Mark Johnson’s image schemata. Popular Music, 18(1), 133-144.

Echard, W. (2006). Sensible virtual selves: Bodies, instruments and the becomingconcrete of Music. Contemporary Music Review, 25(1-2), 7-16.

Feld, S. (1985). Sound and Sentiment: Birds, Weeping, Poetics and Song in Kaluli Expression. Popular Music, 5, 287-289.

Fernández-Viader, M., Moriyón, C., Codorniu i Tomás, I., \& Valdespino, S. (2005). Hacia un estudio lingüístico-contrastivo de la metáfora en dos lenguas de signos: LSE y LSC. In II Congreso Nacional de Lengua de Signos Española (15 al 17 de septiembre de 2005). Valladolid: Universidad de 
Valladolid.

Jacquier, M. de la P., \& Callejas Leiva, D. (2013). Teoría de la metáfora y cognición corporeizada ¿Cómo se introduce la teoría de la metáfora conceptual en los estudios musicales? Epistemus, 2, 51-88.

Johnson, M. L., \& Larson, S. (2003). “Something in the way she moves” Metaphors of musical motion. Metaphor and Symbol, 18(2), 63-84. http://doi.org/10.1207/S15327868MS1802_1

Lakoff, G., \& Johnson, M. (1980). Conceptual metaphor in everyday language. The Journal of Philosophy. http://doi.org/10.2307/2025464

Lakoff, G., \& Johnson, M. (1983). Metaphors We Live by. Language, 59(1), 201. http://doi.org/10.2307/414069

Malamud-Kessler, C., Estañol-Vidal, B., Ayala-Anaya, S., Sentíes-Madrid, H., \& Marco, A. H.-C. (2014). Fisiología de la vibración. Rev Mex Neuroci MayoJunio, 15(3), 163-170.

Moriyón, C., Fernández-Viader, M., \& Codorniu i Tomás, I. (2006). Metáfora y metonimia en dos lenguas de signos: LSE y LSC. In Actes del VII Congrés de Lingüística General (Publicacio). Barcelona.

Moriyón, C., Fuente, M. T., González, N., Martín, S., Rodríguez, G., \& Villameriel, S. (2010). Status quaestionis: la metáfora en la Lengua de Señas Española. In IX Congreso Internacional de Lingüística General (Universidad de Valladolid, 21/23-06-2010).

Nanayakkara, S., Taylor, E., Wyse, L., \& Ong, S. H. (2009). An enhanced musical experience for the deaf: design and evaluation of a music display and a haptic chair. Proceedings of CHI 2009, 337-346.

http://doi.org/10.1145/1518701.1518756

Peñalba, A. (2005). El cuerpo en la música a través de la teoría de la Metáfora de Johnson: análisis crítico y aplicación a la música. Trans: Transcultural Music Review. Revista Transcultural de Música, 9, 8.

Peñalba, A., Moriyón, C., Luque, S., \& Cabezas, G. (2017). La metáfora conceptual como recurso pedagógico musical para personas sordas y con discapacidad auditiva. In Actas del Congreso CNLSE de la Lengua de Signos Española (Real Patro, pp. 87-108). Madrid. 
Platz, F., \& Kopiez, R. (2012). When the eye listens: A meta-analysis of how audiovisual presentation enhances the appreciation of music performance. Music Perception: An Interdisciplinary Journal, 30(1), 71-83.

Saslaw, J. (1996). Forces, containers, and paths: The role of body-derived image schemas in the conceptualization of music. Journal of Music Theory, 40(2), 217-243.

Schraer-Joiner, L. (2014). Music for Children with Hearing Loss: A Resource for Parents and Teachers. Music for Children with Hearing Loss: A Resource for Parents and Teachers.

http://doi.org/10.1093/acprof:osobl/9780199855810.001.0001

Shifres, F. (2007). Poniéndole el cuerpo a la música Cognición corporeizada , movimiento , música y significado. In III Jornadas de Investigación en Disciplinas Artísticas y Proyectuales.

Soria-Urios, G., Duque, P., \& García-Moreno, J. M. (2011). Música y cerebro: fundamentos neurocientíficos y trastornos musicales. Rev Neurol, 52(1), 4555.

Thompson, W. F., Graham, P., \& Russo, F. A. (2005). Seeing music performance: Visual influences on perception and experience. Semiotica, 2005(156), 203227.

Zbikowski, L. M. (2011). Music, language, and kinds of consciousness. In E. Clarke \& D. Clarke (Eds.), Music and Consciousness: Philosophical, Psychological, and Cultural Perspectives (pp. 179-192). Oxford: Oxford University Press. http://doi.org/10.1093/acprof:oso/9780199553792.003.0058 